\title{
The effects of the online gatekeeping using search-based advertising on users' suicidal ideation
}

\author{
Hajime Sueki ${ }^{1 *}$, Asumi Takahashi ${ }^{23}, \&$ Jiro ITO $^{2}$
}

\begin{abstract}
Background: Recently, the practice of "online gatekeeping" related to suicide prevention — placing online advertisements directed at users who search the web for suicide-related terms and leading them to email-based consultations - has been implemented. However, the effectiveness of this practice has not been examined. Aim: To quantitatively examine the effectiveness of suicide-related online gatekeeping. Methods: This was a non-randomized, single-arm study of online gatekeeping among Japanese Internet users. Outcomes were measured at the beginning of the use of the consultation service (T1) and approximately four weeks later (T2). The primary outcome measured was suicidal ideation. Results: In total, 167 participants completed two assessment surveys; approximately $80 \%$ were women, and $59.3 \%$ were young people (in their twenties or younger). Data analysis of 167 online gatekeeping service users showed that the mean suicidal ideation at $\mathrm{T} 2$ was statistically significantly lower than that at $\mathrm{T} 1$, and its effect size was small to medium $(d=0.38)$. Limitations: This study used a single-arm design with no control group. Additionally, the study did not include a second follow-up period. Conclusions: Online gatekeeping is a promising suicideprevention tool, although further research is warranted to examine its effectiveness using a randomized controlled trial.
\end{abstract}

Keywords: suicide prevention, suicidality, suicide risk, internet, web search

\footnotetext{
1 Faculty of Human Sciences, Wako University

2 Specified Nonprofit Corporation OVA

3 Graduate School of Comprehensive Human Sciences, University of Tsukuba

* Corresponding author: Hajime Sueki, Faculty of Human Sciences, Wako University, 5-1-1 Kanaigaoka, Machida, Tokyo, 195-8585, Japan, h_sueki@wako.ac.jp, Tel: +81-44-989-7777.
} 


\section{Introduction}

Every year, 800,000 people worldwide die by suicide, and suicide is one of the leading causes of death, especially among young people (World Health Organization, 2019). Although the evidence for the effectiveness of internet-based intervention for preventing suicide is still insufficient (Zalsman et al., 2016), it is a promising suicide prevention strategy. The promise lies in the potential of the Internet for providing crisis intervention and social support to those at high risk of suicide via an Internet service (e.g., online community, chat, and/or email) (Gilat \& Shahar, 2007; Gilat \& Reshef, 2015), as well as improving their access to health care services (Sueki \& Ito, 2015).

Several studies have been conducted on suicide and the use of web search engines to clarify the behavior of suicidal Internet users (McCarthy, 2010; Yang et al., 2011; Hagihara, 2012; Gunn \& Lester, 2013; Ma-Kellams et al., 2016; Solano et al., 2016; Lee, 2020). These studies have examined the relationship between search volume data for suicide-related words and suicide rates. Many researchers have utilized data published by Google. Although the reliability and validity of Google data are not sufficient (Tran et al., 2017), web searches on suicide have been generally associated with suicide rates. A questionnaire survey of Internet users has also shown an association between the experiences of searching the Web for suicide-related terms and suicidal ideation (Sueki et al., 2014). Additionally, there are some cases of people who died by suicide having searched the Internet for suicide methods just before their suicide attempt (Gunnell et al., 2012). Given the above research findings, it is estimated that Internet users who search the Web for terms such as "suicide methods" are at high risk of suicide.

These behavioral patterns of suicidal Internet users can be used to provide them with crisis intervention and lead them to health care services. Since 2013, suicide prevention strategies that take advantage of web-search characteristics have been conducted in Japan to identify Internet users with a heightened risk of suicide (Sueki \& Ito, 2015). In this suicide prevention practice, an advertisement stating that free email-based consultation is available is displayed on the results' screen of searches for terms such as suicide methods. According to a previous study (Sueki \& Ito, 2018), approximately $80 \%$ of those who saw these ads and sent emails seeking help had suicidal ideation; approximately $40 \%$ had made a suicide attempt in the past. Among the Internet users who sent consultation emails, $28.6 \%$ experienced a positive change in their psychological situation, and $25.3 \%$ adopted a new help-seeking behavior (e.g., visiting a psychiatric hospital) as a result of the consultation.

However, that study (Sueki \& Ito, 2018) did not longitudinally measure the risk of suicide among Internet users who received email-based consultation for suicide prevention; thus, the service's suicide prevention effect is not entirely clear. Therefore, we measured the suicidal ideation 
and depressive/anxiety tendencies of users of an email-based consultation service for suicide prevention at the beginning of their use of the service and about four weeks later to examine its effectiveness.

\section{Methods}

\section{Outline of online gatekeeping}

Figure 1 shows an outline of online gatekeeping activity using search ads. On Google Japan, when a word related to suicide (e.g., suicide methods) was searched, an advertisement displayed on the results screen stating that experts would provide a free consultation. Clicking on the link in the advertisement brought up a web page that outlined the email-based consultation services. The consultation service was free of charge, but those currently undergoing psychiatric treatment were not allowed to use the service and were instructed to go to their doctor's outpatient clinic.

Once the first email from the user was received, the consultation activities were usually initiated within 24 hours. In our first reply, we asked them to complete an online assessment questionnaire survey. At that point, we obtained online informed consent from the service users for the research use of their data. The subsequent consultation activities were mainly based on the Gatekeeper Training Textbook (Japanese Cabinet Office, 2012) developed from the Mental Health First Aid Manual (Kitchener \& Jorm, 2002). The basic procedures for interacting with consultation service users were implemented in the following order: recognizing the target; performing initial approaches (establishing trust-based relationships, supportively listening, and obtaining approval); assessing risks (confirming the presence/absence of suicidal ideation, risk, and protective factors, and attitudes toward problem-solving); and providing support (solving problems and guiding people to social resources such as psychiatric consultation and welfare services). The consultant who replied to the emails was licensed to provide some types of interpersonal support in Japan (e.g., clinical psychologist, psychiatric social worker). Detailed online gatekeeping procedures and instructions for writing a reply email are described in the manual (Specified Nonprofit Corporation OVA, 2020).

\section{Study design \& participants}

This research was conducted using a non-randomized, single-arm study of online gatekeeping for suicide among Japanese Internet users. Figure 2 shows an overview of the study design and recruitment of participants. The study was conducted between 2018 and 2019; the advertisement was displayed 268,381 times and received 5,299 clicks (click rate: 1.97\%). In total, 1023 people sent us emails, and 540 completed an initial assessment questionnaire (T1 survey). There were 184 people who took the first assessment but did not continue the consultation. We asked 327 people who had 
continued to consult with us four weeks after the start of the consultation to answer a questionnaire for a second assessment (T2 survey). A total of 167 people completed the T2 survey (Response rate: $51.1 \%)$.

This study was not pre-registered and we were unable to obtain a priori institutional review board approval because the institution to which the first author belongs has no such board. However, the study was conducted in accordance with the Helsinki Declaration (revised 2013). Prior to participation, potential participants were allowed to quit at any point during the study. We obtained explicit informed consent from the participants online.

\section{Measurements}

The first assessment (T1) was conducted using an online questionnaire survey prior to each participant's initial consultation. The survey consisted of the following items: sex, age, frequency of drinking alcohol, lifetime experience of suicide attempts, lifetime experience of psychiatric hospital visits, suicidal ideation (main outcome), and depression/anxiety tendency (secondary outcome). Approximately one month after the start of the consultation (T2), the participants were asked again about suicidal ideation and depression/anxiety tendency.

Suicidal ideation was measured using the Japanese self-administered Suicidal Ideation Scale (Ohtsuka et al., 1998), based on the Scale for Suicide Ideation designed by Beck, Kovacs, and Weissman (1979). This 13-item instrument was designed to quantify suicidal ideation. Responses are rated on a 3-point scale from 0 to 2 ; higher scores indicate higher suicidal ideation levels (total score range: $0-26$ ). The scale showed good internal consistency (Cronbach's $\alpha=.88$ ), test-retest reliability (3-month interval: $\mathrm{r}=.69$ ), and validity in a survey with 850 internet users (Sueki, 2017).

Depression/anxiety tendency was measured using the K6 scale (Kessler et al., 2002). The K6 is an abridged version of the Kessler Psychological Distress Scale (K10), based on item response theory, to effectively detect mental disorders. For each item, responses were rated on a 5-point scale ranging from 0 to 4 (total score range: $0-24$ ). The Japanese version was developed and has been shown to have the same screening performance as the original (Furukawa et al., 2008).

\section{Statistical analyses}

First, we summarized the demographics of our survey participants. To clarify the characteristics of those who completed the survey in T2, we compared them with those who responded only to the survey in T1. A t-test was employed to compare continuous data, and a chi-square test was used to compare categorical data. 
Second, to examine changes in suicidal ideation and depression/anxiety tendency from the beginning of the consultation to one month later, we conducted a paired t-test with the time of measurement as the independent variable and suicidal ideation and depression/anxiety tendency as the dependent variable and calculated the effect size Cohen's d. For comparison, we also included the results of the largest longitudinal study conducted on suicidal Internet users in Japan (Sueki et al., 2014). This previous study followed suicidal Internet users (e.g., web searches for suicide methods) for six weeks and provided descriptive statistics on suicidal ideation and depression/anxiety tendency measured on the same scale as the present study. We used the data to recalculate effect sizes Cohen's d for longitudinal changes over six week. The test statistics ( $\mathrm{t} \& \mathrm{p}$ ) were cited as reported in the previous study (Sueki et al., 2014). The p-values presented are for twotailed tests, and analysis was performed using SPSS software (SPSS 24.0 for Windows; SPSS Inc., Chicago, IL).

\section{Results}

Table 1 summarizes the participant characteristics. There were 167 participants who completed the $\mathrm{T} 2$ survey and 352 who responded only to the $\mathrm{T} 1$ survey. Those who completed the T2 survey were more likely to be women, less likely to have been to a psychiatric hospital, and had lower suicidal ideation than those who responded only to T1. Approximately $80 \%$ of those who completed T2 were women, 59.3\% were young people in their twenties or younger, and $56.3 \%$ were not in the habit of drinking alcohol. Additionally, $42.5 \%$ had a history of suicide attempts in the past, and $34.1 \%$ had been admitted to a psychiatric hospital at some point. The median number of emails sent by users to the consultants in the approximately four weeks leading up to the T2 survey was 9 , and the median number of emails sent by consultants to users was 10 .

Table 2 summarizes the changes in suicidal ideation from $\mathrm{T} 1$ (the start of consultation) to T2 (one month after the start of consultation). Data analysis of 167 service users of the online gatekeeping showed that the mean suicidal ideation in T2(10.6) was statistically significantly lower than that in T1 (12.6), and its effect size was small to medium, $d=0.38$. The depression/anxiety tendency was also statistically significantly reduced; its effect size was medium, $d=0.50$. A reanalysis of the previous longitudinal study of suicidal internet users (Sueki et al., 2014) showed that suicidal ideation and depression/anxiety tendency were statistically significantly reduced after six weeks. However, the effect size was very small $(d=0.05-0.09)$. 


\section{Discussion}

\section{Principal findings \& comparison with previous studies}

The analysis results indicate that the suicidal ideation and depression/anxiety tendency of the users of email-based online consultation was statistically significantly reduced one month after the start of the consultation, and the effect size was small to medium. A previous study on email-based online consultation (Sueki \& Ito, 2018) reported that $28.6 \%$ of users experienced a positive mood change. However, that finding was based on the results of qualitative analysis of the text from consultation emails. Nevertheless, the results of the qualitative analysis in the previous study were quantitatively supported by this study.

Compared with the results of the longitudinal survey of Japanese Internet users who have used the Internet for suicide-related purposes (e.g., searching the Web for suicide methods, talking to anonymous others about suicidal thoughts) (Sueki et al., 2014), two things could be mentioned about the results of this study. First, those who send emails for consultation via search-linked advertisements have higher suicidal ideation than those who simply use the Internet for suiciderelated purposes. A stronger state of suicidal ideation may encourage help-seeking behavior. Second, suicidal ideation among those using the Internet for suicide-related purposes decreased slightly after six weeks. In contrast, there was a greater reduction in suicidal ideation among users of email-based online consultation, although the period over which we were able to track them was somewhat different. Careful interpretation is necessary due to the different user demographics; however, emailbased online consultations show promise as a type of suicide-prevention intervention.

Although the email-based online consultation may affect suicide prevention, the target population is limited. This study shows that this service is promising when targeted at relatively young women at high risk of suicide because, as has been consistently reported in previous studies (Sueki \& Ito, 2015: 2018), those who send consultation emails via search-linked advertisements for suicide-related terms tend to be young women. Moreover, as seen in this study, men have a lower rate of continuation of consultations, which also decreases the positive effects of consultations. Another previous study also suggests that women are more positively affected by email-based counseling (Gilat \& Reshef, 2015). For men, who are generally at higher risk of suicide mortality than women, it may be necessary to use other intervention techniques (e.g., restricted access to suicide methods) used in combination with counselling for more powerful prevention effects. 


\section{Strengths and limitations}

This study is the first to quantitatively analyze the effects of providing email-based online consultation to Internet users at high risk of suicide after measuring suicidal ideation and depression/anxiety tendency using psychological scales with reliability and validity. However, there are several limitations to this study. First, we used a single-arm design, and there was no control group. Although, similar to the present study, the previous study included a longitudinal survey of Internet users with high suicidal ideation for comparison (Sueki et al., 2014), the demographics of the previous study were different. A randomized controlled trial is needed to more rigorously test the effectiveness of email-based online consultation to Internet users at high risk of suicide. Second, this study did not include a follow-up period, and it is unclear how long the effects of the intervention will last. It is desirable to conduct a follow-up survey to track the users' levels of suicidal ideation over a longer period.

\section{Conclusion and future research}

This study showed that suicidal ideation among users of an email-based online consultation to Internet users at high risk of suicide decreased approximately four weeks after using the service. However, only $51.1 \%$ of the users whose support was still in place at the four-week point responded to the $\mathrm{T} 2$ survey, and this number is not sufficiently high. In general, the cooperation rate for surveys conducted via the Internet is not as high as that for face-to-face surveys; therefore, a different method of evaluation of the psychological state of users in Internet-based consultation services may be required. For example, if a technique for directly estimating suicidal ideation from the text of consultation emails were developed, it would be possible to estimate the psychological state of service users and the degree of suicide risk at any time without conducting a longitudinal questionnaire survey. In order to examine the effectiveness of new forms of suicide prevention practices, it will be necessary to develop new techniques for estimating suicide risk in parallel. 


\section{References}

Beck, A. T., Kovacs, M., \& Weissman, A. (1979). Assessment of suicidal intention: The Scale for Suicide Ideation. Journal of Consulting \& Clinical Psychology, 47(2), 343-352.

https://doi.org/10.1037//0022-006x.47.2.343

Furukawa, T. A., Kawakami, N., Saitoh, M., Ono, Y., Nakane, Y., Nakamura, Y., ... \& Kikkawa, T. (2008). The performance of the Japanese version of the K6 and K10 in the World Mental Health Survey Japan. International Journal of Methods in Psychiatric Research, 17(3), 152158. https://doi.org/10.1002/mpr.257

Gilat, I., \& Reshef, E. (2015). The perceived helpfulness of rendering emotional first aid via email. British Journal of Guidance \& Counselling, 43(1), 94-104. https://doi.org/10.1080/03069885.2014.909006

Gilat, I., \& Shahar, G. (2007). Emotional first aid for a suicide crisis: Comparison between Telephonic hotline and internet. Psychiatry, 70(1), 12-18. https://doi.org/10.1521/psyc.2007.70.1.12

Gunnell, D., Bennewith, O., Kapur, N., Simkin, S., Cooper, J., \& Hawton, K. (2012). The use of the Internet by people who die by suicide in England: A cross sectional study. Journal of Affective Disorders, 141(2-3), 480-483. https://doi.org/10.1016/j.jad.2012.04.015

Gunn III, J. F., \& Lester, D. (2013). Using google searches on the internet to monitor suicidal behavior. Journal of Affective Disorders, 148(2-3), 411-412. https://doi.org/10.1016/j.jad.2012.11.004

Hagihara, A., Miyazaki, S., \& Abe, T. (2012). Internet suicide searches and the incidence of suicide in young people in Japan. European Archives of Psychiatry \& Clinical Neuroscience, 262(1), 39-46. https://doi.org/10.1007/s00406-011-0212-8

Japanese Cabinet. Office (2012). Gatekeeper training textbook. Retrieved February 25, 2021, from http://www8.cao.go.jp/jisatsutaisaku/kyoukagekkan/gatekeeper_text.html

Kessler, R. C., Andrews, G., Colpe, L. J., Hiripi, E., Mroczek, D. K., Normand, S. L., ... \& Zaslavsky, A. M. (2002). Short screening scales to monitor population prevalences and trends in non-specific psychological distress. Psychological Medicine, 32(6), 959-976. https://doi.org/10.1017/S0033291702006074

Kitchener, B. A., \& Jorm, A. F. (2002). Mental health first aid manual. Canberra: Centre for Mental Health Research.

Lee, J. Y. (2020). Search trends preceding increases in suicide: A cross-correlation study of monthly Google search volume and suicide rate using transfer function models. Journal of Affective Disorders, 262, 155-164. https://doi.org/10.1016/j.jad.2019.11.014 
Ma-Kellams, C., Or, F., Baek, J. H., \& Kawachi, I. (2016). Rethinking suicide surveillance: Google search data and self-reported suicidality differentially estimate completed suicide risk. Clinical Psychological Science, 4(3), 480-484.

https://journals.sagepub.com/doi/abs/10.1177/2167702615593475

McCarthy, M. J. (2010). Internet monitoring of suicide risk in the population. Journal of Affective Disorders, 122(3), 277-279. https://doi.org/10.1016/j.jad.2009.08.015

Ohtsuka, A., Seto, M., Kanno, J., \& Agari, I. (1998). Development of the suicide ideation scale for Japanese and a study of the factors related to suicide ideation. Japanese Journal of Counseling Science, 31, 247-258. (Japanese)

Solano, P., Ustulin, M., Pizzorno, E., Vichi, M., Pompili, M., Serafini, G., \& Amore, M. (2016). A Google-based approach for monitoring suicide risk. Psychiatry Research, 246, 581-586. https://doi.org/10.1016/j.psychres.2016.10.030

Specified Nonprofit Corporation OVA (2020). Support Methods for Online Consultation (English Version). Retrieved February 25, 2021, from https://ova-japan.org/?p=6554

Sueki, H., Yonemoto, N., Takeshima, T., \& Inagaki, M. (2014). The impact of suicidality-related internet use: A prospective large cohort study with young and middle-aged internet users. PLOS ONE, 9(4), e94841. https://doi.org/10.1371/journal.pone.0094841

Sueki, H., \& Ito, J. (2015). Suicide prevention through online gatekeeping using search advertising techniques: A feasibility study. Crisis, 36(4), 267-273. https://doi.org/10.1027/02275910/a000322

Sueki, H., \& Ito, J. (2018). Appropriate targets for search advertising as part of online gatekeeping for suicide prevention. Crisis, 39(3), 197-204.https://doi.org/10.1027/0227-5910/a000486

Sueki, H. (2017). Re-examination of reliability and validity of suicide ideation scale. Japanese Journal of Mental Health, 32, 48-54. (in Japanese)

Tran, U. S., Andel, R., Niederkrotenthaler, T., Till, B., Ajdacic-Gross, V., \& Voracek, M. (2017). Low validity of Google Trends for behavioral forecasting of national suicide rates. PLOS ONE, 12(8), e0183149. https://doi.org/10.1371/journal.pone.0183149

World Health Organization. (2019). Suicide in the world: Global health estimates. Retrieved February 25, 2021, from https://www.who.int/teams/mental-health-andsubstance-use/suicide-data

Yang, A. C., Tsai, S. J., Huang, N. E., \& Peng, C. K. (2011). Association of Internet search trends with suicide death in Taipei City, Taiwan, 2004-2009. Journal of Affective Disorders, 132(1-2), 179-184. https://doi.org/10.1016/j.jad.2011.01.019

Zalsman, G., Hawton, K., Wasserman, D., van Heeringen, K., Arensman, E., Sarchiapone, M., ... \& Zohar, J. (2016). Suicide prevention strategies revisited: 10-year systematic review. Lancet Psychiatry, 3(7), 646-659.https://doi.org/10.1016/S2215-0366(16)30030-X 


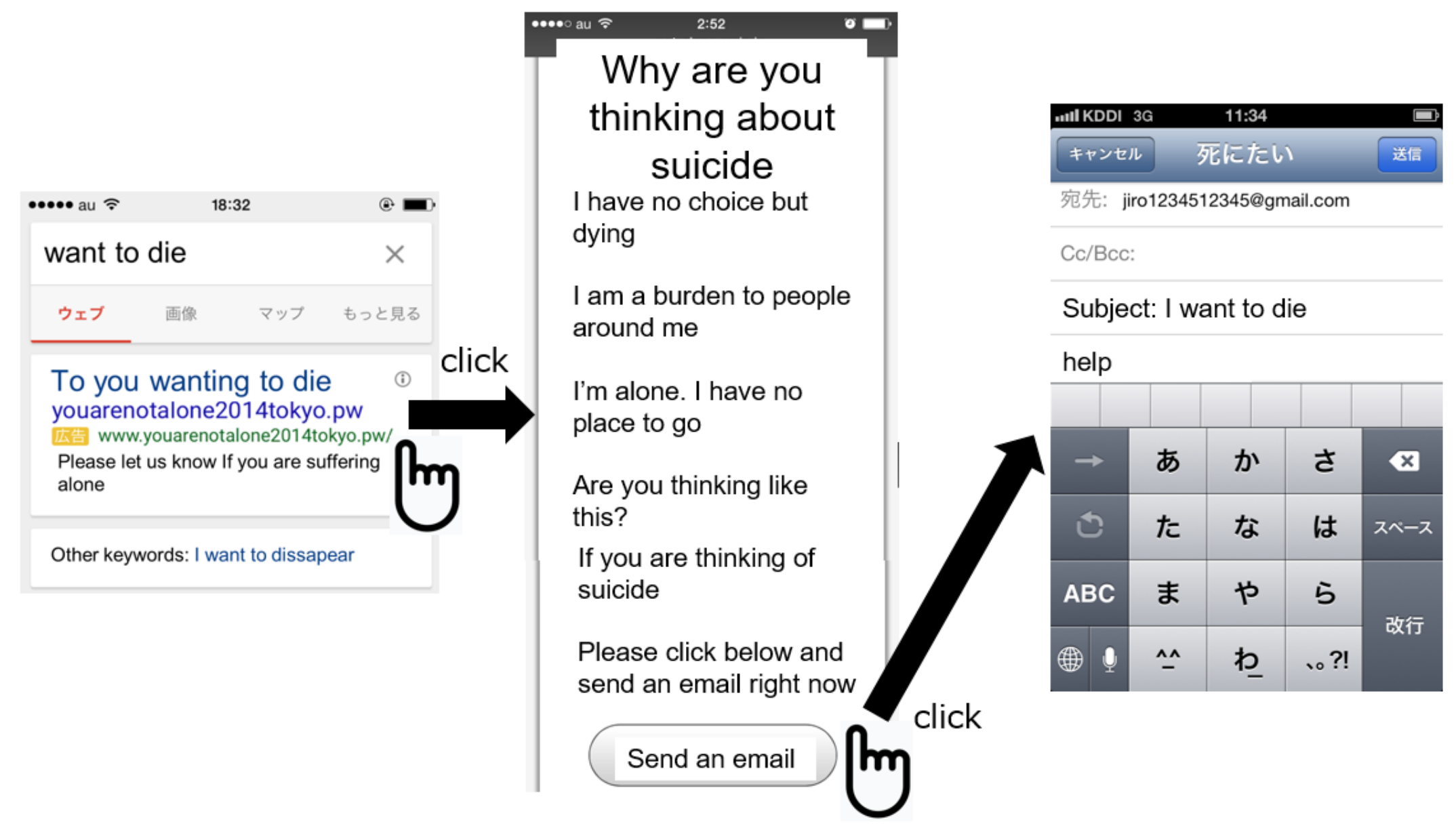

Figure 1

Flow diagram for online gatekeeping 


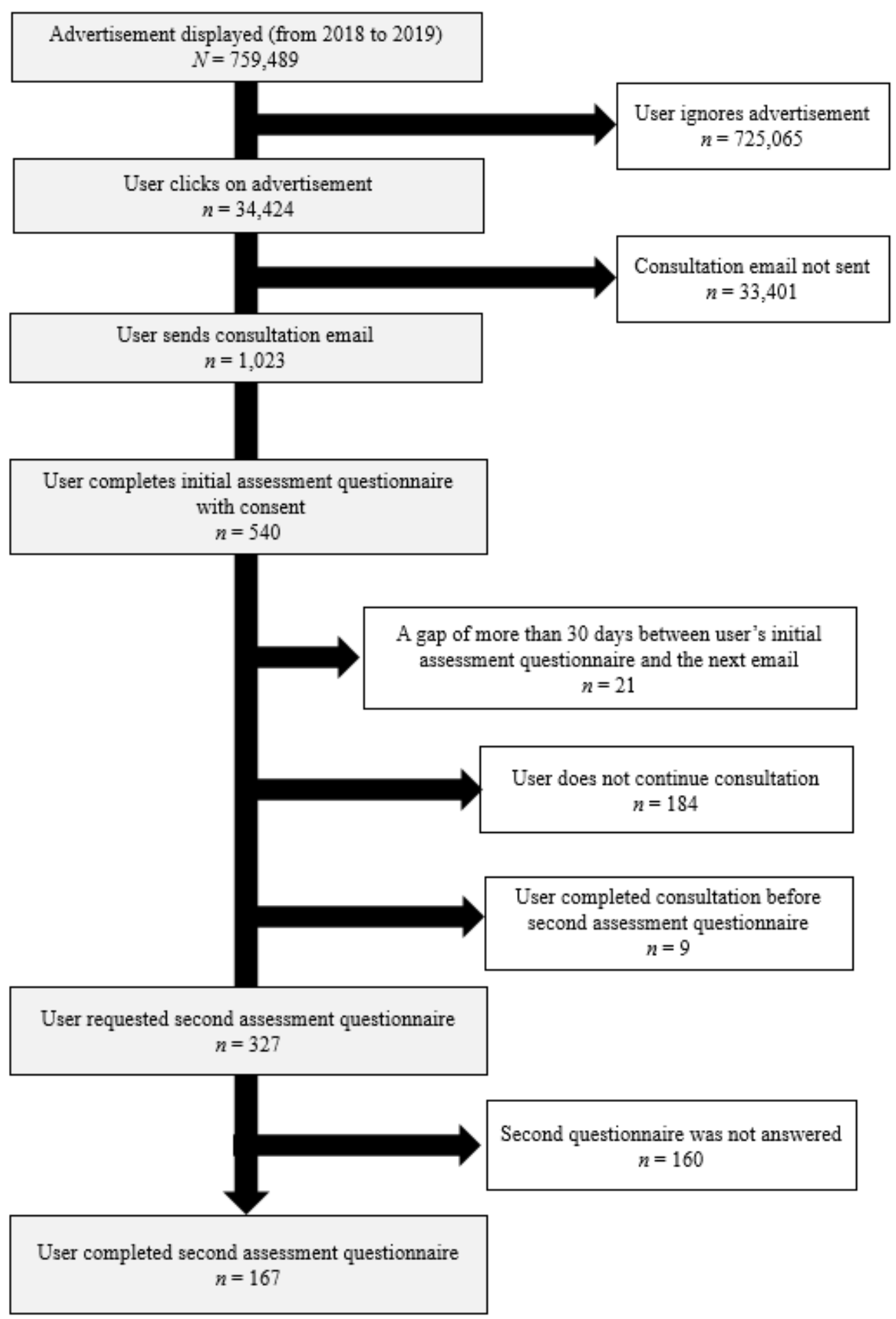

Figure 2

Sampling process 
Table 1.

Descriptive statistics on service users

\begin{tabular}{lccccc}
\hline & \multicolumn{3}{c}{ T2 completed } & Only T1 completed & \\
& $(n=167)$ & $(n=352)$ & $p$ \\
\cline { 2 - 5 } & $n$ & $\%$ & $n$ & $\%$ & \\
\hline Sex; woman & 128 & 76.6 & 234 & 66.5 & 0.047 \\
Age; & & & & & 0.529 \\
10s & 37 & 22.2 & 75 & 21.3 & \\
20s & 62 & 37.1 & 152 & 43.2 & \\
30s & 26 & 15.6 & 54 & 15.3 & \\
40s & 27 & 16.2 & 43 & 12.2 & \\
50s & 12 & 7.2 & 26 & 7.4 & \\
over 60s & 3 & 1.8 & 2 & 0.6 & \\
Frequency of drinking alcohol & & & & & 0.379 \\
Almost never & 94 & 56.3 & 171 & 48.6 & \\
Less than 1 day per week & 38 & 22.8 & 88 & 25.0 & \\
2 to 3 days per week & 15 & 9.0 & 35 & 9.9 & \\
4 to 5 days per week & 4 & 2.4 & 19 & 5.4 & \\
Almost every day & 16 & 9.6 & 39 & 11.1 & \\
Lifetime experience of suicide attempt & 71 & 42.5 & 184 & 52.3 & 0.038 \\
Lifetime experience of psychiatric hospital visit & 57 & 34.1 & 141 & 40.1 & 0.194 \\
Suicidal ideation at T1; Mean SD & 12.6 & 5.1 & 13.5 & 5.1 & 0.065 \\
Depression/Anxiety at T1; Mean SD & 17.9 & 4.0 & 18.0 & 3.8 & 0.866 \\
\hline
\end{tabular}


Table 2.

Changes in suicidal ideation and depression/anxiety tendency among service users and previous study

\begin{tabular}{|c|c|c|c|c|c|c|c|c|c|}
\hline & & \multirow[t]{2}{*}{$N$} & \multicolumn{2}{|c|}{$\begin{array}{l}\text { T1 (At the beginning of } \\
\text { the consultation) }\end{array}$} & \multicolumn{2}{|c|}{ T2 (After about 4 weeks) } & \multirow[t]{2}{*}{$d$} & \multirow[t]{2}{*}{$t$} & \multirow[t]{2}{*}{$p$} \\
\hline & & & Mean & SD & Mean & SD & & & \\
\hline Present & Suicidal ideation & 167 & 12.6 & 5.1 & 10.6 & 5.7 & 0.38 & 6.04 & $<0.001$ \\
\hline \multirow[t]{3}{*}{ Study } & Depression/Anxiety & 167 & 17.9 & 4.0 & 15.3 & 6.3 & 0.50 & 6.15 & $<0.001$ \\
\hline & & $N$ & \multicolumn{2}{|c|}{$\begin{array}{c}\text { T1 (Suicide-related } \\
\text { Internet use) }\end{array}$} & \multicolumn{2}{|c|}{ T2 (After about 6 weeks) } & $d$ & $t$ & $p$ \\
\hline & & & Mean & SD & Mean & SD & & & \\
\hline \multirow{2}{*}{ Sueki et al. (2014) } & Suicidal ideation & 2813 & 8.5 & 5.7 & 8.0 & 5.7 & 0.09 & 8.10 & $<0.001$ \\
\hline & Depression/Anxiety & 2813 & 17.0 & 5.9 & 16.7 & 6.1 & 0.05 & 3.35 & 0.001 \\
\hline
\end{tabular}


The effects of the online gatekeeping for suicide prevention 14 